retains control, no other studio can alter the conditions. The reversing equipment is required at every amplifier point, which may be a simple amplifier station along the line, or a main junction or terminal connecting to a broadcast studio.

\section{Swiss Electrotechnical Industry during War-time}

IN the February issue of Swiss Technics, a journal published by the Swiss Office for the Development of Trade (Zurich and Lausanne), which co-operates with the Swiss Association of Machinery Manufacturers, Zurich, there is an instructive account of a visit made to the Secheron Works at Geneva. These works have always been strictly limited to the construction of heavy electrical machinery and material. A few years ago, when the electrification of the chief Swiss railroads, to which the Secheron Works greatly contributed, drew to a close, new lines of manufacture were tuken up. Among these was the building of electric welding apparatus and the manufacture of the necessary electrodes. The Secheron alternatingcurrent arc-welding apparatus has now almost everywhere replaced direct-current revolving units. For the welding of complicated parts 'exotherme' electrodes are almost universally employed; cut-out or stamped parts can be simply welded together by the melting of the electrode into the space left between them, thus forming a solid block as homogeneous as a foundry casting.

Enormous alternators for the hydro-electric power station of Verbois are now being constructed at the works. These machines resemble two-storied towers, $9 \cdot 2$ metres high and each weighing 300 tons. All parts are electrically welded and every welding has been carefully inspected by radiograph. At normal speed the peripheral speed of the polar wheel will be equal to $155 \mathrm{~km}$. per hour; at $300 \mathrm{rev}$. per min. this speed attains $350 \mathrm{~km}$. per hour. A 3,000-h.p. motor is being built for Norway and twelve transformers of $1,000,2,000$ and $3,200 \mathrm{kva}$. are being built for Ireland.

\section{Electro-formed Reflectors}

THE construction recently of 62 -in. searchlight reflectors at the Bart Laboratories, New Jersey, shows, according to the Electrical Review of April 11, that satisfactory progress is being made in modern electro-forming methods. An electro-formed nickelsurfaced mould is first of all made and a coat of nickel $0.003-0.005 \mathrm{in}$. thick is applied to the outer passivated nickel face. Then follows a coating of copper, to which a pre-formed bronze ring is applied, and the assembly is given a heavy deposit of copper. These firmly bonded layers are stripped from the mould in the form of a bi-metal reflector with copper on the convex face and nickel on the inner polished face. To prevent the introduction of copper into the rhodium bath, a protective mask is fitted on the copper side. The reflector is now ready to receive its rhodium-plating, and in this instance a 'flash' of rhodium 30 millionths of an inch thick appears satisfactory.

The advantage of this method appears to be in the use of rhodium on a nickel base. It has a high hardness (about 600 Brinell), is tightly adherent to its underlying layer, and also has a high degree of reflectivity and resistance to tarnish. The coatings of rhodium used are exceptionally thin; but as nickel is passive when plated with rhodium, adequate protection is provided. It is stated that full protection is lacking with underlayers of copper and its alloys, the relation of which to rhodium in electro-chemical action is such that disfigurement by corrosion may result.

\section{Japan Institute for the Science of Labour}

THE annual report of the Director of the Japan Institute for the Science of Labour for 1939, recently received, in reviewing the present activities of the Institute, emphasizes its objectives in assisting the development of the national industrial capacity, including the training of workers, as well as the improvement of working conditions, and the stimulation of leadership. Reference is made to the investigation. of industrial hazards as well as of the problems of agricultural workers, including their maintenance. A somewhat naive claim is made for the uniqueness of the Institute, as well as a reference to its part in the establishment of a 'New Order' in the Orient. The report also includes the director's address on the "Function and Guiding Spirit of the Institute", given at its first staff conference, in which the character of the Institute as a purely scientific research body covering the daily life of the industrial and agricultural worker is emphasized, and also the importance of its contribution to national productivity and culture. Stress is laid upon the team spirit in the research work of the Institute and its service of social and national needs. Details of the various research sections and laboratories of the Institute are given, as well as of projects already under investigation.

\section{Thin Glass for Microscope Cover-Slips}

THE year 1940 marked the centenary of the manu. facture in Great Britain of specially thin glass for microscope cover-slips. Prior to this date, it was customary for microscopists to use thin talc or mica for covering specimens to be examined under the microscope. This manufacture was started by Messrs. Chance Brothers and Co., Ltd., at Smethwick, in 1840 as nearly as is known, and has been carried on continuously by the same firm up to the present day. The War has called for a considerable increase in supplies of such glass, both in Great Britain and abroad. There was no essential change in this type of glass until about 1928 , when a research was carried out on the development of microscope cover-slips. It was found that the old type of glass, which was essentially like window glass, would not withstand tropical conditions without becoming clouded. Therefore, new methods of manufacturing a glass which would withstand sterilizing and exposure to tropical atmospheres without any deterioration were introduced. The thickness of cover-slip glass is still classified under the headings: Extra thin $(0.075-$ $0.100 \mathrm{~mm}$.$) , No. 1(0 \cdot 100-0 \cdot 167 \mathrm{~mm}$.$) , No. 2(0 \cdot 167$ - 